\title{
Cross reactive immune responses in cattle arising from exposure to Mycobacterium bovis and non- tuberculous mycobacteria
}

Jenkins, $\mathrm{AO}^{1,4}$, Gormley, $\mathrm{E}^{2}$, Gcebe, ${ }^{3}$, Fosgate, $\mathrm{GT}^{4}$, Conan, $\mathrm{A}^{5}$, Aagaard ${ }^{6}, \mathrm{C}$, Michel, $\mathrm{AL}^{4}$, Rutten, $\mathrm{VPMG}^{1,4}$.

\footnotetext{
${ }^{1}$ Division of Immunology, Department of Infectious Diseases and Immunology, Faculty of Veterinary Medicine, Utrecht University, Yalelaan 1, $3584 \mathrm{CL}$, Utrecht, The Netherlands
}

${ }^{2}$ TB diagnostics and Vaccine Research Laboratory, School of Veterinary Medicine, Veterinary Sciences Centre, University College Dublin (UCD), Dublin 4, Ireland

${ }^{3}$ Onderstepoort Veterinary Institute, Agricultural Research Council, Pretoria, South Africa

${ }^{4}$ Department of Veterinary Tropical Diseases, Bovine Tuberculosis and Brucellosis Research Programme, Faculty of Veterinary Science, University of Pretoria, Private Bag X04, Onderstepoort, 0110, South Africa

${ }^{4}$ Department of Production Animal Studies, Faculty of Veterinary Science, University of Pretoria, Private Bag X04, Onderstepoort, 0110, South Africa

${ }^{5}$ Ross University School of Veterinary Medicine, PO Box 334, Basseterre, St Kitts

${ }^{6}$ Department of Infectious Disease immunology, Statens Serum Institut, Artillerivej 5, 2300 Copenhagen, Denmark

\section{Abstract}

Accurate diagnosis of tuberculosis in cattle may be compromised in areas where there are high rates of exposure to environmental /non-tuberculous mycobacteria (NTM). This cross reaction of immune responses to Mycobacterium bovis antigens shared with NTMs can result in reduced specificity of commonly used diagnostic tests including tuberculin skin tests and the interferon gamma assay (IFN४). In this study we assessed the cross-reactive immune responses of $M$. bovis (infected) and NTM 
exposed animals to $M$. bovis and $M$. avium tuberculin, the ESAT6/CFP10 cocktail antigen, tuberculin derived from cultures of selected NTMs, and a panel of recombinant mycobacterium tuberculosis complex (MTBC) antigens sharing homology with orthologues in NTM. Gamma interferon (IFN- $\mathrm{\gamma}$ ) responses were measured in whole blood cultures using the IFN- $\gamma$ assay and the IFN- $\gamma$ elispot assay on purified peripheral blood mononuclear cells (PBMC).

We observed the expected strong IFN- $\gamma$ response to PPD-B in the $M$. bovis infected animals that distinguished this group from non-infected NTM exposed cattle. The IFN- $\gamma$ responses to PPD-N (M. nonchromogenicum), were relatively high in both infected and non-infected NTM exposed cattle, but were not significantly different to classify the true infection status of each group. The results indicated that the cross-reactive responses to PPD-B and/or PPD-A with PPD-N, likely arose from prior exposure to environmental non-tuberculous mycobacteria. The IFN- $y$ immune responses to the 10 R-Mag measured by the IFN- $\gamma$ elispot assay revealed that three of the selected antigens, Rv3615 (ESpC), Rv0287 (esxG) and the ESAT6/CFP10, were immunogenic in the infected cattle, and distinguished the infected cattle from the non-infected NTM exposed animals. The combined data of PPDs and R-Mags derived from NTM mycobacteria may prove useful in future development of novel bTB diagnostic tests.

Keywords: Mycobacterium bovis, tuberculosis, cattle, non-tuberculous mycobacteria, immune responses, diagnostics

\section{$\underline{\text { Introduction }}$}

Exposure of cattle to environmental mycobacteria (NTM) can constrain the detection of $M$. bovis infected animals (bTB) by reducing the specificity of the commonly used diagnostic tests (Gormley, et al., 2013). The false positive cross reactive responses in diagnostic tests are thought to result from exposure of the host to NTM and priming of the host immune system by mycobacterial antigens that 
are shared between NTM and $M$. bovis. To improve the test specificity when screening herds or diagnosing infection in animals, tuberculin derived from M. avium (PPD-A) is often included in the skin test and IFN- - assay as a representative of the NTM. However, in environments where the dominant NTMs are only distantly or not related to M. avium, the choice of PPD-A may not be the most appropriate to gauge specificity. In South Africa, NTM have been isolated from single intradermal comparative tuberculin test (SICTT) positive, or IFN- $\gamma$ reactor animals known to be bTB free (Michel, 2008). For this reason, tuberculin PPD derived from M. fortuitum has also been used as stimulating antigen in the IFN- $\gamma$ assay in South Africa to differentiate between NTM and M. bovis immune responses (Michel, 2008; Michel, et al., 2011). To overcome these difficulties in evaluating specificity, a cocktail of peptides from antigenic proteins, ESAT6 and CFP10, specific for the mycobacterium tuberculosis complex (MTBC) have also been shown useful to enhance the specificity of diagnostic tests (van Pinxteren, et al., 2000). Other recombinant mycobacterial antigens (R-Mags) have been tested in several human and animal studies and also appear to be highly specific for MTBC (Aagaard, et al., 2003; Buddle, et al., 2003; Aagaard, et al., 2004; Aagaard, et al., 2010). The results of whole genome sequencing and proteomic analyses of PPD have revealed that orthologues (homologous genes found in different species) of these R-MAgs are present in a number of NTMs with varying degrees of homology (Aagaard, et al., 2003; Gcebe, et al., 2016; Gcebe and Hlokwe, 2017; Gcebe, et al., 2017). If protein regions in these orthologues share epitopes recognized by the immune system, these may be the source of cross-reactive immune responses in diagnostic tests.

Even with highly specific $M$. bovis antigens (ESAT6 and CFP 10) skin test positive cattle have been identified with cross-reactive responses against M. kansasii, (Vordermeier, et al., 2007). Other antigens related to the Esx-1 secretion system such as Rv3019c (esxR), Rv0287 (esxG) and Rv3615c (espC) are also considered to be highly MTBC specific and immunodominant in bTB infected cattle (Vordermeier, et al., 2005). Another category of antigens considered important for TB diagnosis are the Heat shock proteins (HSP), expressed in conditions of stress and associated with latent infection. One important HSP of Mycobacteria is the HspX protein (Rv2031c) (Sherman, et al., 2001) a 16-kDa 
alpha-crystallin protein. Other antigens of diagnostic relevance and potentially expressed during latent infection are Rv2659c and Rv2660c (Sherman, et al., 2001).

The observation that several NTMs have known protein antigen orthologues with varying levels of homology to MTBC specific antigens prompted us to investigate the cross-reactive immune responses observed in $M$. bovis infected reactor cattle and in non-infected animals exposed to NTM.

\section{Materials and methods}

\section{Selection of $M$. bovis infected and non-infected NTM exposed animals}

The SICTT and collection of blood samples on which the IFN- $\gamma$ tests were carried out was conducted as part of the Irish national bTB eradication programme, which is subject to the EU trade Directive 64/432/EEC, and which governs the nature and frequency of testing. Blood samples were collected under experimental research licences issued by the Department of Health and Children, Republic of Ireland and approved by the UCD Animal Research Ethics Committee (AREC-P-11-49-Gormley). Blood was collected from the jugular vein in lithium-heparin tubes and processed in the laboratory within five hours after collection. The ten naturally infected animals were Holstein Friesian cross breeds aged 1-2 years chosen from heavily tuberculosis - infected herds, and selected based on a positive response to the single intradermal comparative tuberculin test (SICTT), where the skin-fold thickness response to PPD-B exceeded that of PPD-A (Thermo-Fisher Scientific Lelystad, Netherlands) by at least $12 \mathrm{~mm}$. These animals were also positive to the in vitro IFN- $\gamma$ assay on at least two occasions. Following slaughter, the ten cattle underwent a detailed post-mortem examination, in which a range of anatomical sites was examined macroscopically for tuberculosis lesions (including lungs, liver, spleen thoracic lymph nodes, pharyngeal lymph nodes mediastinal lymph nodes, carcase). All ten animals were confirmed positive for tuberculosis by pathology and/or culture of $M$. bovis. The age and breed matched non-infected animals were chosen and purchased from herds with at least a 5- 
year freedom of tuberculosis. These animals were repeatedly test negative on the SICTT and IFN- $\nu$ assay, though chosen on the basis that they generated responses to PPD-A, and where responses to PPD-A > PPD-B in both tests. They were classified negative for bTB following a detailed post-mortem examination. For the purposes of this study, these animals were classified as non-infected but NTM exposed.

\section{Bacteria and preparation of NTM PPD}

Mycobacterium fortuitum (ATCC strain 6841), M. vaccae M. nonchromogenicum and a recently identified strain; M. malmesburyense isolates Gcebe et al., (2017), respectively were grown in 7H9 Middelbrook medium supplemented with OADC (oleic acid, albumin, dextrose, and catalase) (Biolab Diagnostics, Wadeville, South Africa) at a final concentration of $0.1 \%$. The cultures were incubated at $37{ }^{\circ} \mathrm{C}$ for three to four weeks with loosened caps and occasional shaking of the flasks. Before harvesting, the cultures were autoclaved at $121{ }^{\circ} \mathrm{C}$ for $20 \mathrm{~min}$ and filtered through a Whatman 40 filter paper. The culture filtrates were precipitated overnight with $4 \%$ trichloracetic acid (TCA). On the following day the protein precipitate was concentrated by centrifugation (4000 rpm, BeckmanCoulter Allegra X22R) and washed in succession twice with 1\% TCA and once with phosphate buffered saline (PBS), pH 7.2. The concentrated, wet pellet was weighed and dissolved in PBS containing $0.01 \%$ Tween $20, \mathrm{pH} 7.2$, to give a final concentration of $20 \mathrm{mg} / \mathrm{ml}$ (wet weight/volume).

\section{$\underline{\text { Recombinant antigens }}$}

Ten recombinant MTBC antigens (R-Mag) were kindly provided by the Statens Serum Institute (SSI), Denmark (Table 3). Candidate proteins were chosen based on (i) their known orthologues in nontuberculous mycobacteria (all the antigens) (ii) whether they were members of the ESX family or (iii) for their role and relevance in latent TB infections. Of these latter antigens, two have not been commonly reported in animal studies: Rv1120c a conserved hypothetical protein (Sassetti, et al., 
2003) and Rv1284, which was identified by mass spectrometry in the membrane protein fraction and whole cell lysates of M. tuberculosis H37Rv but not in the culture filtrate (de Souza, et al., 2011). a The secreted antigen (CFP20) Rv1932 with thiol peroxidase activity, implicated in intracellular growth of M. tuberculosis, was also included in the panel of antigens.

\section{Data mining of the recombinant antigens}

The presence of the selected antigens in NTM as well as homology of their amino acid sequences relative to H37Rv was determined using protein sequences obtained from bovilist (www.bovilist.com) (Lew, et al., 2011) and subsequent analysis using the NCBI/BLAST/blastp suite (www.blast.ncbi.nlm.nih.gov).

\section{$\underline{\text { ELISpot assay }}$}

Peripheral blood mononuclear cells (PBMCs) were isolated from heparinized blood by Histopaque1077 (Sigma) gradient centrifugation and were immediately cultured in RPMI 1640 tissue culture medium (Sigma) supplemented with 10\% fetal calf serum (Sigma Aldrich), nonessential amino acids (Sigma Aldrich), $5 \times 10^{-5} \mathrm{M}$ 2-mercaptoethanol, $100 \mathrm{U} / \mathrm{ml}$ penicillin, and $100 \mu \mathrm{g} / \mathrm{ml}$ streptomycin sulfate. Secretion of IFN- $\gamma$ by stimulated peripheral blood mononuclear cells was assessed in the enzyme-linked immunosorbent spot (ELISPOT) assay as described by Vordermeier et al., (2007).

\section{Interferon gamma release assays}

Blood samples from $M$. bovis infected and non-infected cattle were tested for IFN- $\gamma$ using the Bovigam assay (Thermo Fisher Ltd, Switzerland) according to the manufacturer's instructions. Heparinized whole blood of each animal was dispensed into wells of a sterile 24-well tissue culture tray $(1.0 \mathrm{ml} /$ well, 3 wells for each animal) and stimulated with $20 \mathrm{ug} / \mathrm{ml}$ of PPD-B, PPD-A, the 4 NTM 
PPD (PPD-F, PPD-M, PPD-N, and PPD-V), the hybrid recombinant molecule ESAT6/CFP10 was used at $5 \mathrm{ug} / \mathrm{ml}$, pokeweed mitogen at $5 \mu \mathrm{g} / \mathrm{ml}$ as positive control, and PBS as negative control. The supernatant of each well was collected after culture for 20 to $24 \mathrm{~h}$ at $37^{\circ} \mathrm{C}$ in $5 \% \mathrm{CO}_{2}$ and tested for the presence of IFN- $\gamma$. Cattle with PPD-B-simulated blood plasma were considered infected when PPD-B - PPD-A was $\geq 0.1$ OD (de la Rua-Domenech, et al., 2006).

\section{Statistical methods}

The sample size for the study was determined from an independent population of $M$. bovis infected $(\mathrm{N}=20$ and non-infected NTM exposed $(\mathrm{N}=20)$ animals (see: Selection of $M$. bovis infected and noninfected NTM exposed animals). The calculation (epitools.ausvet.com.au) was based on the sample size required to detect a statistically significant difference in the means between the two groups by measuring IFN- $\gamma$ responses to PPD-B tuberculin (Mean OD of infected animals: 2.3. Mean OD of NTM exposed animals: 0.46). There was an assumption of unequal variances (Var of infected animals: 1.024. Var of NTM exposed animals: 0.585 ) and allowance for unequal sample sizes between groups. Confidence levels were set at 0.95 and Power level at 0.8 . Sample size required was estimated as eight $M$. bovis infected and four NTM exposed animals. The 10 infected animals and 5 NTM exposed study animals were selected from similar independent population of animals.

Animals were classified as bTB positive in the IFN- $\gamma$ diagnostic assay when the differences $(\Delta)$ between immune responses to PPD-B and PPD-A was $\geq 0.1$ ( $\triangle$ PPD B-PPD A $\geq 0.1$ ) and NTM exposed otherwise ( $\triangle P P D$ B-PPD A $<0.1$ ). Differences in immune responses to NTM PPDs (including PPD-A), PPD-B as well as ESAT6/CFP10 in the infected and NTM exposed animals were assessed for normality by calculating descriptive statistics and performing the Shapiro-Wilk normality test. These data violated the normality assumption and were therefore descriptively presented using boxplots and dot plots (results of each individual animal presented for the stimulations with different 
recombinant proteins). The non-parametric Mann-Whitney test was used to test for statistically significant responses of PPD antigens between bTB infected and NTM exposed cattle. The Kruskal -Wallis tests for analysis of variance $(\alpha<0.5)$ with Dunn's post hoc multiple comparison test and adjusted $p$ value was used to compare the IFN- $-\gamma$ responses generated by the PPD antigens and RMag antigens. Statistical analysis was carried out using Graphpad Prism 6.0g (GraphPad Software Inc).

$\underline{\text { Results }}$

\section{IFN- $\gamma$ responses to PPDs from MTBC and NTM mycobacteria}

To evaluate immune responses of bTB infected and NTM exposed animals to different mycobacterial antigens of MTBC and NTM origin, we stimulated whole blood with a panel of antigens including PPD $A$ and $B$, and PPDs prepared from selected NTMs and measured IFN- $\gamma$ responses. The ESAT6/CFP10 cocktail antigen was also used as a monitor of diagnostic specificity (Fig. 1). The infection status of M. bovis infected and NTM exposed animals was clearly distinguished by significant differences in the responses to PPD-b $(p=0.02,95 \% \mathrm{Cl}$ of difference: $0.5-3.1)$ and ESAT6/CFP10 $(p=0.0007,95 \%$ $\mathrm{Cl}$ of difference: $0.25-1.8)$. Among the panel of NTM PPDs, the highest median responses were recorded with PPD $\mathrm{N}$ in both groups, while the lowest responses were measured with PPD-F and PPD-M. There were no differences measured in the responses to NTM PPDs between the $M$. bovis infected and NTM exposed animals with the exception of PPD-V, which was borderline higher in the infected group ( $\mathrm{P}=0.09,95 \% \mathrm{Cl}$ of difference: $-0.08-1.40)$.

Comparison of all the PPDs using ANOVA indicated significant differences in the M. bovis infected ( $p$ $<0.0001)$ and NTM exposed $(P<0.0007)$ animals. Post-hoc multiple pairwise comparisons revealed 
that there were few significant differences among the PPDs, and these were more frequent in the $M$. bovis infected cattle (Table 2).

\section{Diagnostic interpretation of IFN- $\gamma$ responses to NTM PPDs}

In order to compare the responses to NTM PPDs and PPD-A as a measure of diagnostic test performance, the differences in IFN- $\gamma$ responses between PPD-B and each of the NTM PPDs (including PPD A) were calculated (Table 1). The bTB positive status of skin test reactor animals was defined by the Bovigam results when PPD-B - PPD-A was $\geq 0.1$ OD. We also interpreted that an animal was positive in the IFN- $\gamma$ test for $b T B$ when the response of PPD B - PPD NTM was $\geq 0.1$ OD. Using PPD-A as the reference NTM antigen, all of the skin test bTB positive animals were classified as true positive and all skin test negative animals were true negative. When PPD-N was used instead of PPD-A, all of skin test negative animals remained as classified true negative, and all but one of the skin test positive animals were classed as true positive (Table 1). Conversely, when comparing the remaining NTM PPDs i.e. PPD-F, PPD-M, PPD-N, PPD-V, we observed that the differential immune responses between PPD-B and those NTM PPDs were equal to or greater than 0.1 OD in both the skin test negative animals as also in the skin test positive infected animals. This indicated a lower specificity using this test interpretation and the inability to use the IFN- $\gamma$ responses of these NTM PPDs to correctly classify the infection status of the animals.

Evaluation of the IFN-y responses to MTBC specific recombinant antigens using the ELISpot assay

In the next study, we measured IFN-y responses following antigen stimulation of PBMC from $M$. bovis infected and NTM exposed animals with 10 recombinant mycobacterial antigens (R-Mag) of MTBC origin (including the ESAT6/CFP10 cocktail). The responses to recombinant antigens were expressed as total spot forming units (SFU) using an IFN-y ELISpot assay (Figure 2). One of the NTM 
exposed cattle was excluded from the analysis due to a high background responses. Among all of the antigens tested, the median IFN-y responses to three antigens, Rv3615 ( $p=0.008), \operatorname{Rv287}(p=0.008)$ and ESAT 6/CFP $10(p=0.004)$ were significantly higher in infected animals compared with the controls. Comparison of the antigens using ANOVA indicated significant differences in the responses among in the $M$. bovis infected $(p<0.0001)$ but not in the NTM exposed $(P=0.8)$ animals. Pairwise multiple comparisons of responses to the antigens in the infected animals revealed that Rv287 and ESAT6/CFP10 generated significantly higher responses than most of the remaining antigens. There was no significant difference in the responses of animals to antigen stimulation with Rv287 and ESAT6/CFP10.

\section{Comparison of protein sequence homologies of MTBC antigens in common NTMs}

The percentage levels of homology in amino acid sequences between the nine H37Rv R-Mag and ESAT6/CFP10 and their presence in 5 NTM used for IFN-y testing, as well as those most abundant in South Africa, were obtained from the NCBI database and are highlighted (in bold) in Table 3 . ESAT6/CFP10 was used as a hybrid molecule in the in vitro assays but the individual antigens were considered in this sequence data analysis. The frequencies of $M$ tuberculosis $\mathrm{H} 37 \mathrm{Rv}$ proteins that are also among the coding genes of the 9 NTM included were; ESAT6: 9/9, CFP10: 9/9, Rv2031: 8/9, Rv3615c: 4/9, Rv2660c: 3/9, Rv2659c: 3/9, Rv3019c: 7/9, Rv1932: 7/9, Rv1120C:8/9, Rv0287: 8/9, Rv1283: 6/9.

\section{Discussion}


When immune sensitization in cattle occurs by natural exposure to environmental NTMs, this can pose problems for diagnosis of bTB if there are sufficient cross-reactive responses to compromise the performance of the skin test and the IFN- $\gamma$ diagnostic assay. The inclusion of the PPD-A in the routine diagnostic tests for bovine tuberculosis is aimed at improving the specificity of the diagnostic tests by minimising the proportion of non-infected animals responding as false positives to PPD-B. These test false positive animals are assumed to have been exposed to particular NTM, which share antigens with the members of the mycobacteria tuberculosis complex. The PPD-A is used as a representative of the diverse population of NTMs that may be present in any locale, and is primarily used because of its shared antigenic repertoire with $M$. bovis. The comparative in vitro immune reactivity to PPD-B and PPD-A in non-infected but NTM exposed animals may differ depending on the level of exposure to any particular NTM that shares common antigens with $M$. bovis. The nature of these cross-reactive mycobacterial responses between $M$. bovis and NTM is not well understood. In this study, we set to evaluate bovine immune responses following antigen stimulation of blood samples from known bTB infected and non-infected NTM exposed cattle to PPDs derived from NTM and also to a panel of recombinant MTBC antigens.

Our results showed that whole blood stimulation with NTM PPDs generated IFN- $\gamma$ responses in both infected and non-infected animals. Apart from the expected dominant PPD-B response in the bTB infected animals, which distinguished infected from non-infected animals, only one other antigen (PPD-V) generated responses that were borderline higher in the infected animals. This could indicate that there are unique antigens present in $M$. vaccae that are recognized by $M$. bovis infected cattle or, more likely, that there is a high degree of homology beween $M$. bovis specifc antigens and related antigens in $M$. vaccae. It is also plausible to suggest that differing levels of homology between antigens present in $M$. bovis and those NTM species may have been responsible for the variable degree of cross reactivity measured, with respect to both the magnitude of the response and number of reactor animals that responded. Likewise, the responses to PPD-B observed in the NTM exposed animals was likely the result of immune priming by NTM sharing homologous epitopes 
with M. bovis antigens. This is consistent with the very low IFN- $\gamma$ responses recorded following blood stimulation of the NTM exposed cattlewith $M$. bovis specific ESAT6/CFP10 antigens compared to broad recognition of multi-antigen PPD B.

Another finding from study was the measurement of relatively strong and variable responses in the infected and NTM exposed cattle to PPD derived from M. nonchromogenicum (PPD-N). However, there were no significant differences found in the IFN- $\gamma$ responses to stimulation with PPD-B, PPD-A and PPD-N in the infected or in the NTM exposed animals, Although natural exposure of the bTB infected cattle to $M$. nonchromogenicum followed by a specific immune response cannot be ruled out (McCorry et al. 2004), our results suggest that the immune response towards PPD-N may have arisen from cross reactive immune responses between $M$. bovis and/or $M$. avium, and $M$. nonchromogenicum. Functional activity originating from cross reactive immune responses between M. vaccae and M. bovis BCG has been reported in a previous study (Demangel, et al., 2005), where mice exposed and sensitized to $M$. vaccae prior to BCG vaccination were better protected than nonsensitized mice in an experimental tuberculosis model.

The production of MTBC specific recombinant antigens has facilitated developments to improve the specificity of diagnostic tests (Buddle, et al., 1999; Buddle, et al., 2001; Buddle, et al., 2003; Casal, et al., 2012; Xin, et al., 2013). The rationale is that these antigens will only be recognized by infected hosts. However, the true specificity of some of these recombinant antigens has been questioned because of the presence of related orthologues in NTM (Xin, et al., 2013), potentially compromising the promise of high specificity. In order to gain a better understanding we selected nine recombinant MTBC specific molecules with known orthologues in NTM (R-Mag). The homologies in AA sequences varied from $25 \%$ to $98 \%$ comparing H37Rv and their orthologues in the nine different NTMs.

The IFN- $\gamma$ immune responses to the R-Mag measured in vitro indicated that three of the selected antigens, Rv3615 (EspC), Rv0287 (esxG) and the ESAT6/CFP10, were immunogenic in infected cattle, 
and distinguished the infected cattle from the non-infected NTM exposed animals. In the NTM exposed animals, no significant differences in IFN- $\gamma$ responses were found following stimulation with the R-Mag. The Rv0287 (esxG) and Rv3615c (espC) have previously been shown to be highly MTBC specific and immunodominant in bTB infected cattle (Vordermeier, et al., 2005). Despite R-Mag homology of $43 \%, 74 \%$ and $40 \%$ in $M$. avium, M. nonchromogenicum and $M$. vaccae, respectively, with the 2031c antigen of $M$. tuberculosis H37Rv strain, exposure to NTM did not result in detectable immune responses to this R-Mag in the NTM exposed animals. In addition, the $M$. tuberculosis H37Rv 0287 antigen homologous at $85 \%$ and $76 \%$ levels with orthologues in $M$. avium and $M$. vaccae did also not induce immune responses in NTM exposed cattle. Since PBMC of NTM exposed cattle did not show responses to the selected R-Mag, we could not determine a relationship between cross-reactivity and the degree of homology of antigens between the selected NTM and M. bovis. We concluded from these findings that the R-Mag showed variable degrees of immunogenicity in the context of exposure to $M$. bovis, but they did not appear to be immunogenic following NTM exposure.

In extrapolating the results of this study to a wider population of infected and disease free herds we recognize that the degree of cross-reactivity measured may have been influenced by biased selection of the cattle. The animals were pre-selected based on their highly biased PPD bovine > PPD avian measurements in the skin test and IFN- $\gamma$ responses in the infected group. In the NTM group, there was a bias towards PPD-A > PPD-B. This was necessary to maximize the likelihood of detecting and comparing the cross-reactive responses. This pilot study does not reveal how these antigens might respond in less biased groups and additional testing will be required in routine breakdown herds to gauge the potential usefulness of including these PPDs and R-Mags in any future diagnostic test.

In conclusion, we have demonstrated cross-reactive immune responses to selected NTM PPDs in cattle infected with $M$. bovis. We have also shown that non-infected but NTM reactor animals 
generate immune responses to PPD-B in common with NTM PPDs. We conclude that NTM exposure may predispose the immune response in cattle to potentially bias bTB diagnosis. Depending on the degree of homology in antigens shared between $M$. bovis and NTM, variable levels of cross-reactive immune responses might be established following exposure to specific mycobacteria (either $M$. bovis or NTM). This can be detected following in vitro stimulation with antigens from the particular group of mycobacteria (NTM or M. bovis). Our results highlight the potential diagnostic relevance of Rv3615 and Rv287, and emphasize the need for continued evaluation of ESAT6/CFP10 and conventional PPD antigens.

\section{References}

Aagaard, C., Brock, I., Olsen, A., Ottenhoff, T.H., Weldingh, K., Andersen, P., 2004. Mapping immune reactivity toward Rv2653 and Rv2654: Two novel low-molecular-mass antigens found specifically in the mycobacterium tuberculosis complex. J. Infect. Dis. 189, 812-819.

Aagaard, C., Govaerts, M., Meikle, V., Gutierrez-Pabello, J.A., McNair, J., Andersen, P., SuarezGuemes, F., Pollock, J., Espitia, C., Cataldi, A., 2010. Detection of bovine tuberculosis in herds with different disease prevalence and influence of paratuberculosis infection on PPDB and ESAT-6/CFP10 specificity. Prev. Vet. Med. 96, 161-169.

Aagaard, C., Govaerts, M., Meng Okkels, L., Andersen, P., Pollock, J.M., 2003. Genomic approach to identification of mycobacterium bovis diagnostic antigens in cattle. J. Clin. Microbiol. 41, 3719-3728.

Betts, J.C., Lukey, P.T., Robb, L.C., McAdam, R.A., Duncan, K., 2002. Evaluation of a nutrient starvation model of mycobacterium tuberculosis persistence by gene and protein expression profiling. Mol. Microbiol. 43, 717-731. 
Buddle, B.M., McCarthy, A.R., Ryan, T.J., Pollock, J.M., Vordermeier, H.M., Hewinson, R.G., Andersen, P., de Lisle, G.W., 2003. Use of mycobacterial peptides and recombinant proteins for the diagnosis of bovine tuberculosis in skin test-positive cattle. Vet. Rec. 153, 615-620.

Buddle, B.M., Ryan, T.J., Pollock, J.M., Andersen, P., de Lisle, G.W., 2001. Use of ESAT-6 in the interferon-gamma test for diagnosis of bovine tuberculosis following skin testing. Vet. Microbiol. 80, $37-46$.

Buddle, B.M., Parlane, N.A., Keen, D.L., Aldwell, F.E., Pollock, J.M., Lightbody, K., Andersen, P., 1999. Differentiation between mycobacterium bovis BCG-vaccinated and M. bovis-infected cattle by using recombinant mycobacterial antigens. Clin. Diagn. Lab. Immunol. 6, 1-5.

Casal, C., Bezos, J., Diez-Guerrier, A., Alvarez, J., Romero, B., de Juan, L., Rodriguez-Campos, S., Vordermeier, M., Whelan, A., Hewinson, R.G., Mateos, A., Dominguez, L., Aranaz, A., 2012. Evaluation of two cocktails containing ESAT-6, CFP-10 and rv-3615c in the intradermal test and the interferon-gamma assay for diagnosis of bovine tuberculosis. Prev. Vet. Med. 105, 149-154.

de la Rua-Domenech, R., Goodchild, A.T., Vordermeier, H.M., Hewinson, R.G., Christiansen, K.H., Clifton-Hadley, R.S., 2006. Ante mortem diagnosis of tuberculosis in cattle: A review of the tuberculin tests, gamma-interferon assay and other ancillary diagnostic techniques. Res. Vet. Sci. 81, 190-210.

de Souza, G.A., Arntzen, M.Ø., Fortuin, S., Schürch, A.C., Målen, H., McEvoy, C.R.E., van Soolingen, D., Thiede, B., Warren, R.M., Wiker, H.G., 2011. Proteogenomic analysis of polymorphisms and gene annotation divergences in prokaryotes using a clustered mass spectrometry-friendly database. Molecular \& Cellular Proteomics 10, 
Demangel, C., Garnier, T., Rosenkrands, I., Cole, S.T., 2005. Differential effects of prior exposure to environmental mycobacteria on vaccination with mycobacterium bovis BCG or a recombinant BCG strain expressing RD1 antigens. Infect. Immun. 73, 2190-2196.

Gcebe, N., Hlokwe, T.M., 2017. Non-tuberculous mycobacteria in south african wildlife: Neglected pathogens and potential impediments for bovine tuberculosis diagnosis. Front. Cell. Infect. Microbiol. 7, 15.

Gcebe, N., Michel, A., Gey van Pittius, N.C., Rutten, V., 2016. Comparative genomics and proteomic analysis of four non-tuberculous mycobacterium species and mycobacterium tuberculosis complex: Occurrence of shared immunogenic proteins. Front. Microbiol. 7, 795.

Gcebe, N., Rutten, V., Pittius, N.G.V., Naicker, B., Michel, A., 2017. Mycobacterium malmesburyense sp. nov., a non-tuberculous species of the genus mycobacterium revealed by multiple gene sequence characterization. Int. J. Syst. Evol. Microbiol. 67, 832-838.

Gormley, E., Doyle, M., Duignan, A., Good, M., More, S.J., Clegg, T.A., 2013. Identification of risk factors associated with disclosure of false positive bovine tuberculosis reactors using the gammainterferon (IFNgamma) assay. Vet. Res. 44, 117-9716-44-117.

Lew, J.M., Kapopoulou, A., Jones, L.M., Cole, S.T., 2011. TubercuList--10 years after. Tuberculosis (Edinb) 91, 1-7.

Michel, A.L., 2008. Mycobacterium fortuitum infection interference with mycobacterium bovis diagnostics: Natural infection cases and a pilot experimental infection. J. Vet. Diagn. Invest. 20, 501503. 
Michel, A.L., Cooper, D., Jooste, J., de Klerk, L.-., Jolles, A., 2011. Approaches towards optimising the gamma interferon assay for diagnosing mycobacterium bovis infection in african buffalo (syncerus caffer). Prev. Vet. Med. 98, 142-151.

Sassetti, C.M., Boyd, D.H., Rubin, E.J., 2003. Genes required for mycobacterial growth defined by high density mutagenesis. Mol. Microbiol. 48, 77-84.

Sherman, D.R., Voskuil, M., Schnappinger, D., Liao, R., Harrell, M.I., Schoolnik, G.K., 2001. Regulation of the mycobacterium tuberculosis hypoxic response gene encoding alpha -crystallin. Proc. Natl. Acad. Sci. U. S. A. 98, 7534-7539.

van Pinxteren, L.A., Ravn, P., Agger, E.M., Pollock, J., Andersen, P., 2000. Diagnosis of tuberculosis based on the two specific antigens ESAT-6 and CFP10. Clin. Diagn. Lab. Immunol. 7, 155-160.

Vordermeier, H.M., Brown, J., Cockle, P.J., Franken, W.P., Drijfhout, J.W., Arend, S.M., Ottenhoff, T.H., Jahans, K., Hewinson, R.G., 2007. Assessment of cross-reactivity between mycobacterium bovis and M. kansasii ESAT-6 and CFP-10 at the T-cell epitope level. Clin. Vaccine Immunol. 14, 1203-1209.

Vordermeier, H.M., Pontarollo, R., Karvonen, B., Cockle, P., Hecker, R., Singh, M., Babiuk, L.A., Hewinson, R.G., van Drunen I-van Den Hurk,S., 2005. Synthetic peptide vaccination in cattle: Induction of strong cellular immune responses against peptides derived from the mycobacterium bovis antigen Rv3019c. Vaccine 23, 4375-4384.

Xin, T., Jia, H., Ding, J., Li, P., Yang, H., Hou, S., Yuan, W., Guo, X., Wang, H., Liang, Q., Li, M., Wang, B., Zhu, H., 2013. Assessment of a protein cocktail-based skin test for bovine tuberculosis in a doubleblind field test in cattle. Clinical and Vaccine Immunology 20, 482-490. 

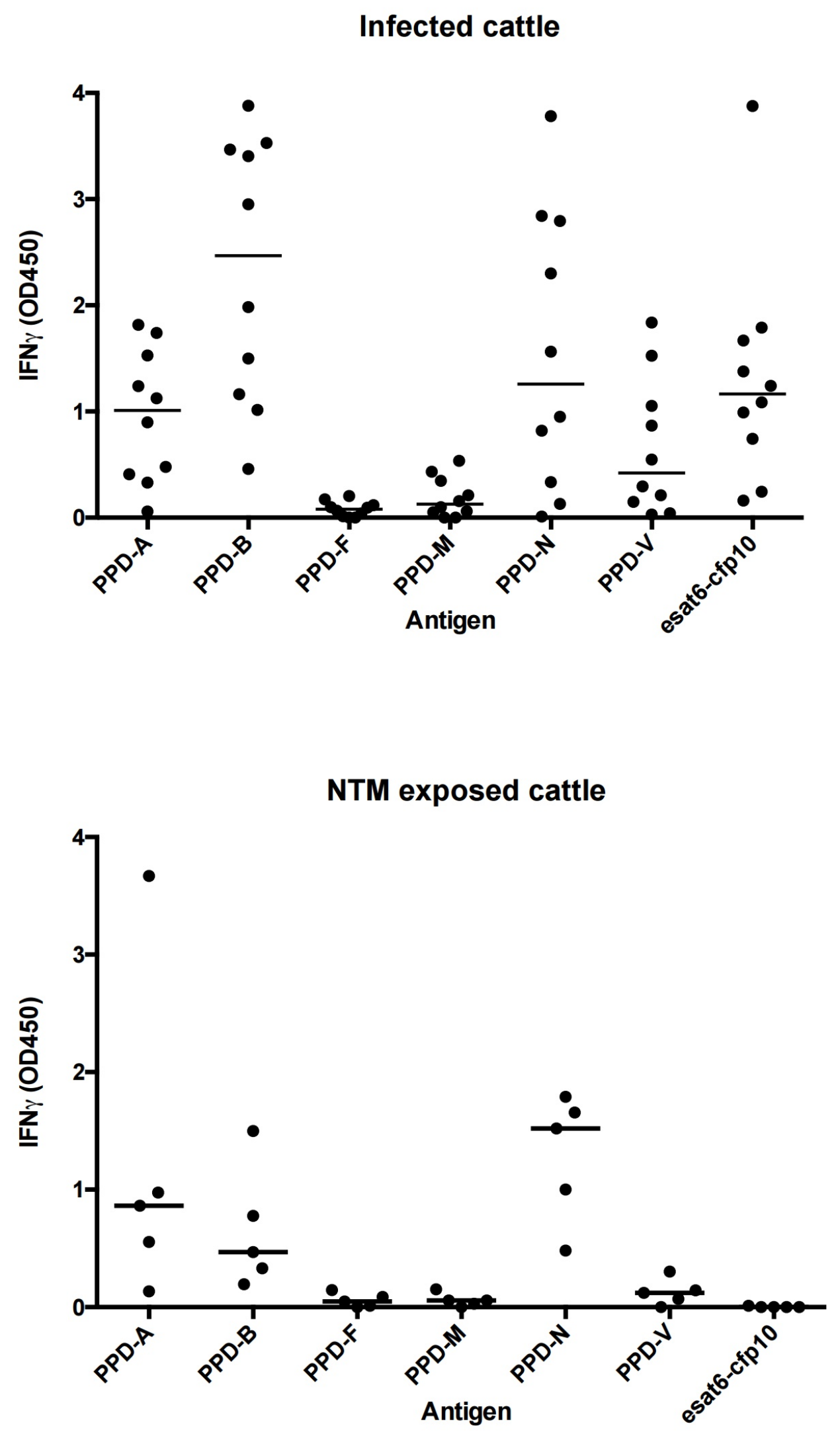


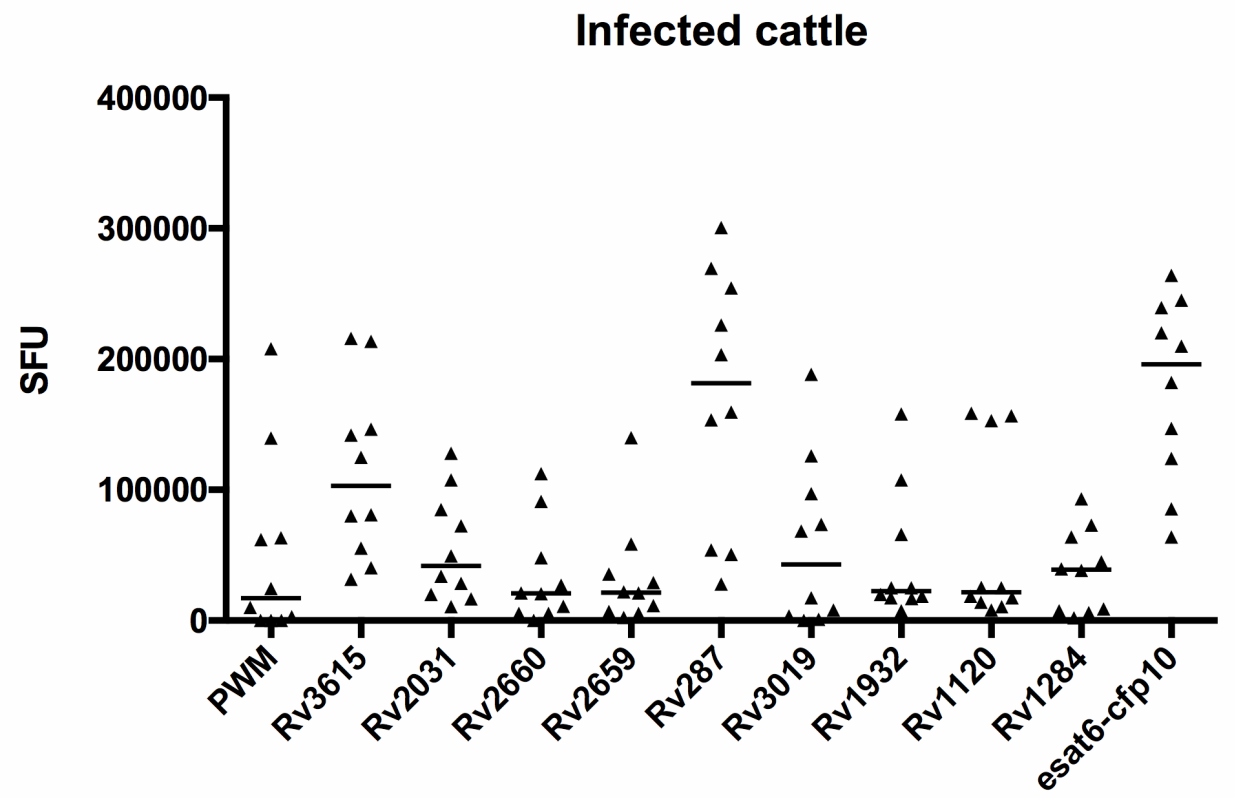

Antigens

\section{Control cattle}

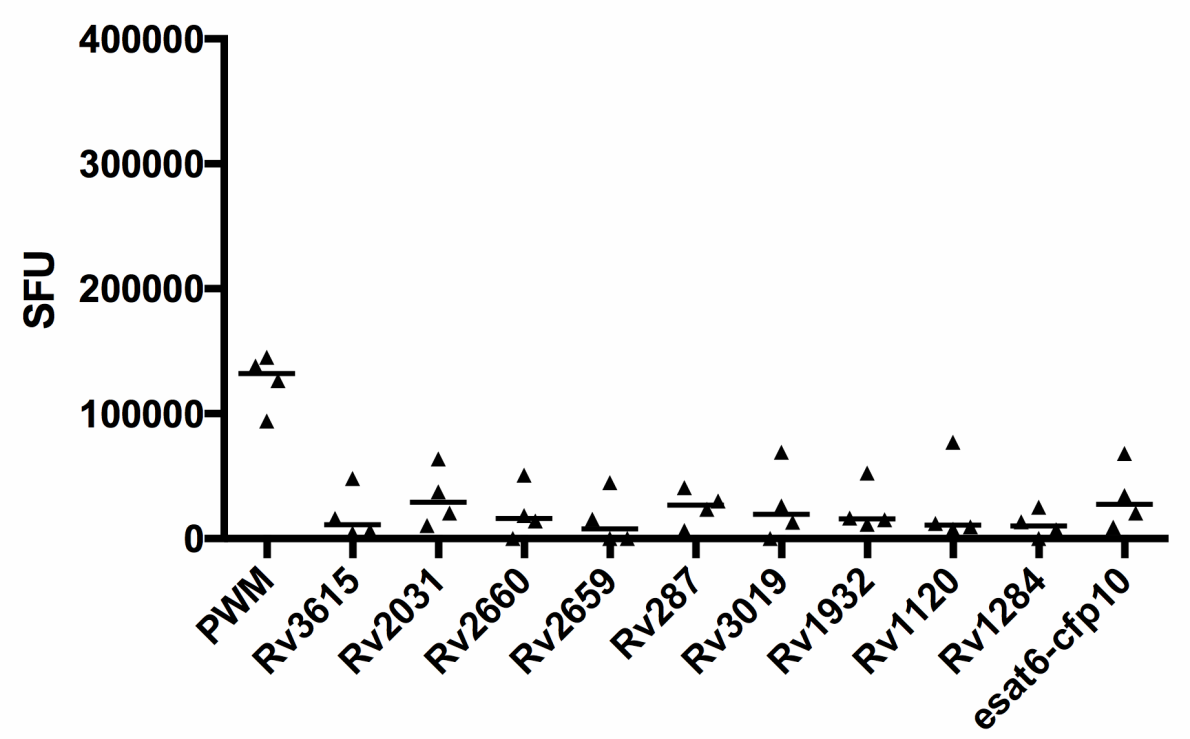

Antigens 
Fig. 2: ELISpot interferon-gamma responses induced by stimulation of peripheral blood mononuclear cells with 10 different recombinant Mycobacterium bovis antigens in 10 bovine tuberculosis (bTB) infected cattle and four NTM exposed cattle. The SFU values are expressed per $\mathrm{ml}$ i.e. $\times 1000$. Each dot represents the responses from an individual animal. Median values for each of the animal responses to the antigens are indicated by a line.

Table 1: Comparison of the diagnostic potential of the NTM PPDs $F, M, N$ and $V$ with the routine PPD A. $\triangle$ PPD B-PPD NTM represents the difference between PPD and the respective NTM PPD. The differences PPD B minus PPD A (all > 0.1) in positive animals are indicated by the underlined values

\begin{tabular}{lccccccc}
\hline Skin test & ID & PPD B & $\boldsymbol{\Delta}$ B-A & $\boldsymbol{\Delta}$ B-F & $\boldsymbol{\Delta}$ B-M & $\boldsymbol{\Delta}$ B-N & $\Delta$ B-V \\
negative & 1 & 1.49 & -2.17 & 1.36 & 1.44 & -0.29 & 1.38 \\
negative & 2 & 0.77 & -0.08 & 0.69 & 0.63 & -0.88 & 0.48 \\
negative & 3 & 0.19 & 0.06 & 0.21 & 0.19 & -0.29 & 0.19 \\
negative & 4 & 0.46 & -0.51 & 0.42 & 0.41 & -1.05 & 0.33 \\
negative & 6 & 0.33 & -0.22 & 0.32 & 0.3 & -0.67 & 0.26 \\
positive & 7 & 2.95 & $\underline{1.21}$ & 2.75 & 2.52 & 0.16 & 1.9 \\
positive & 8 & 1.01 & $\underline{\underline{0.95}}$ & 1.02 & 1.02 & 0.68 & 0.97 \\
positive & 9 & 1.98 & $\underline{1.08}$ & 1.89 & 1.77 & 0.42 & 1.43 \\
positive & 10 & 1.49 & $\underline{1.02}$ & 1.46 & 1.4 & 0.55 & 1.2 \\
positive & 11 & 1.16 & $\underline{\underline{0.75}}$ & 1.18 & 1.18 & 1.03 & 1.01 \\
positive & 13 & 3.87 & $\underline{2.76}$ & 3.87 & 3.83 & 1.04 & 2.04 \\
positive & 14 & 3.52 & $\underline{2.29}$ & 3.36 & 2.99 & -0.25 & 2 \\
positive & 15 & 3.40 & $\underline{1.59}$ & 3.35 & 3.25 & 2.59 & 3.2 \\
positive & 16 & 3.46 & $\underline{1.94}$ & 3.35 & 3.12 & 1.17 & 2.6 \\
\hline
\end{tabular}

Table 2: Pairwise comparison of Bovigam responses to antigens in infected and NTM exposed animals

\begin{tabular}{|l|c|c|}
\hline \multicolumn{3}{|c|}{ Adjusted P value } \\
& Infected & NTM exposed \\
\hline PPD-A vs. PPD-B & $>0.99$ & $>0.99$ \\
\hline PPD-A vs. PPD-F & $0.0371^{*}$ & 0.11 \\
\hline PPD-A vs. PPD-M & 0.34 & 0.15 \\
\hline PPD-A vs. PPD-N & $>0.99$ & $>0.99$ \\
\hline PPD-A vs. PPD-V & $<0.0001^{*}$ & 0.53 \\
\hline PPD-B vs. PPD-F & & \\
\hline
\end{tabular}




\begin{tabular}{|c|c|c|}
\hline PPD-B vs. PPD-M & $0.0006 *$ & 0.35 \\
\hline PPD-B vs. PPD-N & $>0.99$ & $>0.99$ \\
\hline PPD-B vs. PPD-V & 0.16 & $>0.99$ \\
\hline PPD-F vs. PPD M & $>0.99$ & $>0.99$ \\
\hline PPD-F vs. PPD-N & $0.0032 *$ & $0.012^{*}$ \\
\hline PPD-F vs. PPD-V & 0.33 & $>0.99$ \\
\hline PPD-M vs. PPD-N & $0.0473 *$ & $0.016^{*}$ \\
\hline PPD-M vs. PPD-V & $>0.99$ & $>0.99$ \\
\hline PPD-N vs. PPD-V & $>0.99$ & 0.08 \\
\hline
\end{tabular}

Comparisons were done with Wilcoxon non-parametric test to test the difference in population mean ranks between the different combinations of pairs of antigens in bTB infected animals and NTM exposed animals. $\left({ }^{*}\right)$ represents comparisons when $(p \leq 0.05)$. The antigen in bold letters is the antigen with the higher immune response value for each significant comparison. 
Table 3: Recombinant antigens (R-Mag) used in this study, presence of their genes in in Mycobacterium spp. relevant to this study and their relative homologies to $M$. tuberculosis $\mathrm{H} 37 \mathrm{Rv}$

\begin{tabular}{|c|c|c|c|c|c|c|c|c|c|c|}
\hline $\begin{array}{l}\text { Syno } \\
\text { nym }\end{array}$ & $\begin{array}{l}\text { Position } \\
\text { on } \\
\text { M.tub } \\
\text { H37Rv }\end{array}$ & $\begin{array}{l}\text { (\%) } \\
\text { Acc.no }\end{array}$ & $\begin{array}{l}\text { M. } \\
\text { fortuitum } \\
\text { (\%) } \\
\text { Acc. no }\end{array}$ & $\begin{array}{l}\text { M. } \\
\text { nonchro } \\
m . \\
(\%) \\
\text { Acc. no }\end{array}$ & $\begin{array}{l}\text { M. } \\
\text { morio } \\
k . \\
\text { (\%) } \\
\text { Acc no }\end{array}$ & $\begin{array}{l}\text { (\%) } \\
\text { Acc. no }\end{array}$ & $\begin{array}{l}\text { (\%) } \\
\text { Acc. no }\end{array}$ & $\begin{array}{l}\text { (\%) } \\
\text { Acc. no }\end{array}$ & $\begin{array}{l}\text { M. marinum } \\
\text { (\%) } \\
\text { Acc. no }\end{array}$ & $\begin{array}{l}\text { M. } \\
\text { szulgai } \\
\text { (\%) } \\
\text { Acc. no }\end{array}$ \\
\hline $\begin{array}{l}\text { ESAT } \\
6 \\
\text { esxA }\end{array}$ & Rv3875 & $\begin{array}{l}28 \\
\text { ETB47313 } \\
.1\end{array}$ & $\begin{array}{l}2 \\
\text { WP_00388 } \\
3250\end{array}$ & & & $\begin{array}{l}28 \\
\text { WP_00392 } \\
9216\end{array}$ & $\begin{array}{l}72 \\
\text { WP_00389 } \\
1394.1\end{array}$ & $\begin{array}{l}97 \\
\text { ACG70854. } \\
1\end{array}$ & $\begin{array}{l}95 \\
\text { WP_020727 } \\
043.1 \\
\end{array}$ & $\begin{array}{l}85 \\
\text { ACF475 } \\
72.1 \\
\end{array}$ \\
\hline $\begin{array}{l}\text { CFP1 } \\
0 \\
\text { esxB }\end{array}$ & Rv3874 & $\begin{array}{l}25 \\
\text { ETZ55332 } \\
.1 \\
\end{array}$ & $\begin{array}{l}64 \\
\text { WP_00388 } \\
3249.1\end{array}$ & & & $\begin{array}{l}41 \\
\text { WP_00393 } \\
0721\end{array}$ & $\begin{array}{l}61 \\
\text { WP_00389 } \\
1393.1\end{array}$ & $\begin{array}{l}95 \\
\text { WP_02336 } \\
8929.1\end{array}$ & $\begin{array}{l}97 \\
\text { WP_020727 } \\
678.1 \\
\end{array}$ & $\begin{array}{l}93 \\
\mathrm{ACH} 88 \\
457.1 \\
\end{array}$ \\
\hline $\begin{array}{l}\text { TB } \\
16.2\end{array}$ & $\begin{array}{l}\text { Rv2031 } \\
\text { c }\end{array}$ & $\begin{array}{l}43 \\
\text { EUA4096 } \\
2.1\end{array}$ & $\begin{array}{l}98 \\
\text { AAM } 69225 \\
.1\end{array}$ & $\begin{array}{l}74 \\
\text { AAM692 } \\
071\end{array}$ & & $\begin{array}{l}40 \\
\text { WP_00393 } \\
0275\end{array}$ & $\begin{array}{l}59 \\
\text { WP_01530 } \\
8029.1\end{array}$ & $\begin{array}{l}82 \\
\text { EUA22056. } \\
1\end{array}$ & $\begin{array}{l}73 \\
\text { AAM69211.1 }\end{array}$ & \\
\hline $\mathrm{ESpC}$ & $\begin{array}{l}\text { Rv3615 } \\
\text { c }\end{array}$ & & & & & $\begin{array}{l}32 \\
\text { WP_00393 } \\
1047\end{array}$ & $\begin{array}{l}27 \\
\text { WP_00389 } \\
7859.1\end{array}$ & $\begin{array}{l}67 \\
\text { WP_02336 } \\
8576.1\end{array}$ & $\begin{array}{l}65 \\
\text { WP_012395 } \\
741.1\end{array}$ & \\
\hline$N A$ & $\begin{array}{l}\text { Rv2660 } \\
\text { c }\end{array}$ & $\begin{array}{l}29 \\
\text { WP_0197 } \\
37419\end{array}$ & & & & & $\begin{array}{l}\mathbf{5 7} \\
\text { WP_01530 } \\
6581.1\end{array}$ & $\begin{array}{l}32 \\
\text { WP_00389 } \\
6598.1\end{array}$ & & \\
\hline NA & $\begin{array}{l}\text { Rv2659 } \\
\text { c }\end{array}$ & $\begin{array}{l}\text { WP_0238 } \\
68494\end{array}$ & & & & & $\begin{array}{l}28 \\
\text { WP_00389 } \\
7599.1\end{array}$ & $\begin{array}{l}63 \\
\text { WP_02337 } \\
2703.1 \\
\end{array}$ & $\begin{array}{l}\mathbf{5 6} \\
\text { CDM76125.1 }\end{array}$ & \\
\hline $\begin{array}{l}\text { TB } \\
10.3 \\
\text { esxR }\end{array}$ & $\begin{array}{l}\text { Rv3019 } \\
\text { c }\end{array}$ & $\begin{array}{l}80 \\
\text { YP_88398 } \\
8.1\end{array}$ & $\begin{array}{l}73 \\
\text { WP_00388 } \\
0383 .\end{array}$ & & & $\begin{array}{l}68 \\
\text { WP_00393 } \\
3734\end{array}$ & $\begin{array}{l}72 \\
\text { WP_00389 } \\
2041.1 \\
\end{array}$ & $\begin{array}{l}86 \\
\text { EUA16504. } \\
1\end{array}$ & & \\
\hline $\begin{array}{l}\text { CFP } \\
20 \\
\text { tpx }\end{array}$ & Rv1932 & $\begin{array}{l}31 \\
\text { YP_88088 } \\
7 .\end{array}$ & $\begin{array}{l}84 \\
\text { WP_00388 } \\
4841\end{array}$ & & & $\begin{array}{l}82 \\
\text { WP_00393 } \\
2421\end{array}$ & $\begin{array}{l}85 \\
\text { WP_00389 } \\
4924.1\end{array}$ & $\begin{array}{l}85 \\
\text { WP_02336 } \\
4353.1\end{array}$ & $\begin{array}{l}88 \\
\text { WP_020729 } \\
103.1\end{array}$ & \\
\hline $\mathrm{NA}$ & $\begin{array}{l}\text { Rv1120 } \\
\text { c }\end{array}$ & $\begin{array}{l}81 \\
\text { ETB54823 }\end{array}$ & $\begin{array}{l}58 \\
\text { WP_00388 } \\
4198\end{array}$ & & & $\begin{array}{l}51 \\
\text { WP_00393 } \\
1622\end{array}$ & $\begin{array}{l}70 \\
\text { WP_01530 } \\
8806.1\end{array}$ & $\begin{array}{l}85 \\
\text { EUA11197. } \\
1\end{array}$ & $\begin{array}{l}84 \\
\text { WP_020726 } \\
328.1\end{array}$ & \\
\hline $\begin{array}{l}\text { TB } \\
9.8 \\
\text { esxG }\end{array}$ & Rv0287 & $\begin{array}{l}85 \\
\text { WP_0238 } \\
69950\end{array}$ & $\begin{array}{l}81 \\
\text { WP_00388 } \\
0382\end{array}$ & & & $\begin{array}{l}76 \\
\text { WP_00393 } \\
3735.1\end{array}$ & $\begin{array}{l}76 \\
\text { WP_01172 } \\
7071.1\end{array}$ & $\begin{array}{l}91 \\
\text { WP_02336 } \\
9870.1\end{array}$ & $\begin{array}{l}86 \\
\text { WP_012394 } \\
694.1\end{array}$ & \\
\hline $\operatorname{can} A$ & Rv1284 & $\begin{array}{l}80 \\
\text { WP_0238 } \\
65461\end{array}$ & $\begin{array}{l}78 \\
\text { WP_00388 } \\
5327\end{array}$ & & & & $\begin{array}{l}78 \\
\text { WP_00389 } \\
6391.1\end{array}$ & $\begin{array}{l}83 \\
\text { EUA13307. } \\
1\end{array}$ & $\begin{array}{l}85 \\
\text { WP_020730 } \\
007.1\end{array}$ & \\
\hline
\end{tabular}

**Empty cells indicate absence of information regarding the NTM on the NCBI database. 